\title{
Adjuvant immunotherapy
}

DOI:

10.1016/j.ejca.2020.03.016

\section{Document Version}

Accepted author manuscript

Link to publication record in Manchester Research Explorer

\section{Citation for published version (APA):}

Higham, C. E., Chatzimavridou-Grigoriadou, V., Fitzgerald, C. T., Trainer, P. J., Eggermont, A. M. M., \& Lorigan, P. (2020). Adjuvant immunotherapy: the sting in the tail. European Journal of Cancer, 132, 207-210.

https://doi.org/10.1016/j.ejca.2020.03.016

\section{Published in:}

European Journal of Cancer

\section{Citing this paper}

Please note that where the full-text provided on Manchester Research Explorer is the Author Accepted Manuscript or Proof version this may differ from the final Published version. If citing, it is advised that you check and use the publisher's definitive version.

\section{General rights}

Copyright and moral rights for the publications made accessible in the Research Explorer are retained by the authors and/or other copyright owners and it is a condition of accessing publications that users recognise and abide by the legal requirements associated with these rights.

\section{Takedown policy}

If you believe that this document breaches copyright please refer to the University of Manchester's Takedown Procedures [http://man.ac.uk/04Y6Bo] or contact uml.scholarlycommunications@manchester.ac.uk providing relevant details, so we can investigate your claim.

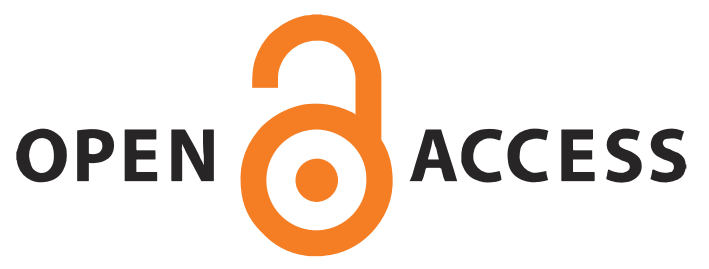




\section{Adjuvant immunotherapy: the sting in the tail.}

Claire E. Higham ${ }^{1}$, Viktoria Chatzimavridou-Grigoriadou ${ }^{1}$, Cheryl T. Fitzgerald ${ }^{2}$, Peter J. Trainer $^{1}$, Alexander M.M. Eggermont $\mathrm{AMM}^{3}$, Paul Lorigan ${ }^{4}$

1 Department of Endocrinology, Christie Hospital NHS Foundation Trust, Manchester, University Of Manchester, Manchester Academic Health Science Centre, Manchester, UK

2 Department of Reproductive Medicine, Manchester University NHS Foundation Trust, Manchester, University of Manchester, Manchester Academic Health Science Centre, Manchester, UK

3 Princess Máxima Center for Pediatric Oncology, Heidelberglaan 25, 3584 CS, Utrect, Netherlands

4 Institute of Cancer Sciences, University of Manchester and The Christie NHS Foundation Trust, Manchester, UK

Corresponding author: paul.lorigan@christie.nhs.uk

\section{Abstract}

Adjuvant therapy with PD-1 inhibitors for resected Stage III/IV melanoma reduce the risk of recurrence by $40-50 \%$ and is now a standard of care. Immune related adverse events (IRAEs) occurred in approximately $37 \%$ of patients in the pivotal trials, $10-15 \%$ were severe (Grade 3-4). Endocrine toxicities were common and mostly irreversible. Thyroid toxicity occurred in $15-20 \%$ of patients, hypophysitis (2.2\%), insulin dependent diabetes mellitus (1\%) and adrenalitis (1\%). Revision of the AJCC staging system (Version 8) has resulted in a significant improvement in prognosis for patients with Stage III disease. As a result, clinicians may now offer adjuvant immunotherapy to patients with a lower risk of recurrence than those in the pivotal trials. There is a need to balance the relatively small reduction of absolute risk of recurrence against the risk and impact of toxicity. Five-ten percent of biochemically euthyroid patients on levothyroxine report symptoms of depression. Hypogonadism can result from toxicity to the hypothalamic-pituitary axis, and can lead to sexual dysfunction and subfertility. Secondary hypogonadism can be treated by the administration of FSH and $\mathrm{LH}$ which induce spermatogenesis/ovulation in a functioning gonad, but is not always successful. Insulin dependent diabetes mellitus (DM) often presents with rapid onset of 
hyperglycemia and potentially life-threatening diabetic ketoacidosis. Long term adverse outcomes are likely to mimic Type 1 DM with a 6-fold increase in cardiovascular disease related mortality and 3-fold in all-cause mortality. These survivorship issues are relevant to all melanoma patients, but articularly pertinent where the absolute benefit is modest.

Key words: adjuvant immunotherapy, melanoma, endocrine toxicity, fertility, late effects

Checkpoint inhibitors have revolutionised the outcomes for patients in a number of different tumour types, with a 5-year survival of $>50 \%$ recently reported for metastatic melanoma ${ }^{1}$. The unparalleled benefits seen in metastatic disease have led to this approach being evaluated in the adjuvant setting. The CTLA-4 inhibitor ipilimumab is associated with a survival benefit of $10 \%$ in resected stage III disease when compared to placebo or to high dose interferon, but treatment related toxicity has limited its use..$^{2.3,4}$ Two pivotal trials of adjuvant PD-1 inhibitors (pembrolizumab in resected Stage $\mathrm{II}^{5}$ and nivolumab in resected Stage III and IV disease patients ${ }^{6}$ ) showed a reduction in risk of relapse of $40-50 \%$, survival data have not yet been reported, studies of adjuvant PD-1 inhibitor are ongoing in earlier stage melanoma (NCT03553836, NCT03405155) and in other tumour types. The toxicity profiles for pembrolizumab and nivolumab are well established in the metastatic setting, and there were no new or unexpected toxicities reported in the adjuvant setting. Immune related adverse events (IRAEs), directly related to the mechanism of action of the drugs, occurred in approximately $37 \%$ of patients, and in $10-15 \%$ of patients these were severe (Grade 3-4). Endocrine toxicities were common in both studies and were irreversible for most patients. Thyroid toxicity was the most prevalent, occurring in approximately $15-20 \%$ of patients. Other important toxicities include hypophysitis (2.2\%), insulin dependent diabetes mellitus (1\%) and adrenalitis (1\%). A recent meta-analysis indicated a fatality rate of $5.5 \%$ of patients presenting with acute hypoadrenalism secondary to immunotherapy.

As a result of the significant reduction in risk of melanoma recurrence, pembrolizumab and nivolumab were approved for adjuvant use by the FDA and EMA in resected high-risk Stage 
III disease and nivolumab in resected Stage IV, and are now in routine clinical practice. Importantly, whilst both studies recruited only higher risk Stage III patients (microscopic nodal disease $>1 \mathrm{~mm}$ for pembrolizumab, Stage IIIB or higher for nivolumab), as defined by the American Joint Committee on Cancer (AJCC) staging system Version 7, the approved indication was all Stage III patients. Furthermore, around the time of approval, revision of the AJCC staging system (Version 8$)^{7}$ resulted in a significant improvement in prognosis for patients with Stage III disease, as a result of which clinicians may now offer adjuvant immunotherapy to patients with a lower risk of recurrence than those enrolled in the pivotal trials. Such patients have a very good prognosis without treatment. The expected 10 -year survival for a patient with Stage IIla disease is $88 \%$, hence there is an increased need to balance the relatively small reduction of absolute risk of recurrence against the risk and impact of toxicity.

Primary hypothyroidism occurred in up to $15 \%$ of patients in the pivotal trials. Autoimmune hypothyroidism in the general population is considered to be a benign condition. However, $5-10 \%$ of biochemically euthyroid patients on levothyroxine report symptoms of depression and impaired mental wellbeing. ${ }^{8}$ Increased mortality has been observed in the very small number of patients who do not achieve biochemical euthyroidism on replacement, with an increased risk of cardiovascular-related mortality when TSH persistently exceeds $10 \mathrm{mIU} / \mathrm{L}^{9}$

Hypoadrenalism most commonly results from adrenocorticotropin hormone (ACTH) deficiency secondary to hypophysitis, with underlying adrenalitis being a less frequent cause. A recent meta-analysis indicated a fatality rate of $5.5 \%$ of patients presenting with acute hypoadrenalism secondary to immunotherapy ${ }^{10}$. Hypothalamic-Pituitary-Adrenal (HPA) axis damage following immunotherapy is virtually always irreversible and life-long glucocorticoid therapy is necessary. ${ }^{11}$ Whilst this can be effectively managed with input and education from specialist Endocrine teams it is not without risk; Scandinavian and European registries describe mortality associated with all cause adrenal insufficiency as $1.5-3$ fold that of the general population. ${ }^{12,13}$ Long-term glucocorticoid replacement therapy carries the risk of complications associated with over-treatment and impaired quality of life. Hypophysitis 
can result in isolated ACTH deficiency or panhypopituitarism, with the latter potentially requiring additional life-long hormonal replacement therapy. ${ }^{11}$

For many younger patients, potential effects on future fertility are of concern. Hypogonadism can result from toxicity to the hypothalamic-pituitary axis, and can lead to sexual dysfunction and subfertility. It is unknown whether immunotherapy causes direct gonadal damage. Although sex steroid replacement can be offered to control symptoms in both groups, success of fertility treatment will be dependent on gonadal integrity. Hypogonadism secondary to immunotherapy induced hypophysitis can be treated by the administration of FSH and $\mathrm{LH}$ which induce both spermatogenesis and ovulation in a functioning gonad. Induction of spermatogenesis is a highly effective treatment particularly for men who have undergone normal pubertal development prior to their hypogonadotropic condition. ${ }^{14}$ However, it is time consuming and costly, and often assisted conception treatments are required. Semen storage is both easy and of low cost, and many centres undertake this routinely before adjuvant immunotherapy where male patients are concerned about potential loss of fertility.

Ovulation induction with gonadotropins is also a highly effective fertility treatment ${ }^{15}$ leading to pregnancy rates of approximately $25 \%$ per cycle for women with hypogonadotropic hypogonadism. As with all fertility treatments, chance of success reduces significantly with advancing female age. The treatment involves daily injections of gonadotrophins and must be monitored carefully with ultrasound scanning to ensure that there is not an overresponse. Although complex, the treatment is more straightforward than assisted conception treatments, allowing couples to achieve pregnancy through intercourse once ovulation has been triggered. Multiple cycles are possible leading to high cumulative pregnancy rates, although pregnancy is not guaranteed. If a pregnancy does not occur, hyperstimulation of the ovaries with gonadotropins can be used for assisted conception treatment, such as IVF. There are currently no data on whether immunotherapy can result in primary hypogonadism. As a result, there is no consensus on the role of fertility preservation in this setting, particularly in women. Oocyte cryopreservation is time 
consuming, costly and still relatively unsuccessful. ${ }^{16}$ So whilst there are fertility options available for patients affected with hypogonadotropic hypogonadism, the treatments are relatively invasive, costly and are frequently not successful. Funding for fertility treatment is usually subject to restriction based on several eligibility criteria meaning that many patients will need to self-fund treatment.

Insulin dependent diabetes mellitus (DM) has been linked to PD-1/ PD-L1 inhibitors with a reported incidence of around 1\%; 5,6 it often presents with rapid onset of hyperglycemia and potentially life-threatening diabetic ketoacidosis (DKA). Additionally, there is little evidence of reversibility to checkpoint inhibitor induced beta-cell destruction resulting in a life-long dependence on insulin replacement. ${ }^{17}$ Adverse outcomes from the resultant diabetes are likely to mimic Type $1 \mathrm{DM}$ in which there is an augmentation of microvascular disease and 6fold increase in cardiovascular disease (CVD) related mortality and 3-fold in all-cause mortality. ${ }^{18}$

The added cost implications of toxicity in the long term are also an important consideration. For example, conservative estimates of annual costs relating to the management of Type 1 DM and adrenal insufficiency in the US are over $\$ 5000$ and between $\$ 10-20,000$ respectively. ${ }^{19,20}$

Emerging developments in tissue based molecular predictors, circulating biomarkers and the microbiome may help predict which patients who are 'low risk' by conventional staging are more likely to relapse or develop certain toxicities. Furthermore, there is emerging evidence that development of immune toxicities, in particular endocrinopathies, is associated with better overall survival ${ }^{21}$, suggesting that targeting treatment to those with less chance of developing the toxicity may be associated with reduced efficacy. 
Better understanding of the long-term complication of immunotherapy will require extended follow-up of Pharma sponsored pivotal trials, registry studies and a focus on biomarkers of toxicity. Whilst the potential absolute survival benefit from adjuvant immunotherapy treatment reduces in lower risk patients, the risk of developing toxicity remains the same. Accurate estimation and effective communication of the potential survival benefit of immunotherapy needs to be set in the context of potential long-term mortality, morbidity and cost of toxicities. These survivorship concerns span all tumour types and are not limited to the lower risk patients but are an even greater challenge in this group.

1 Larkin J, Chiarion-Sileni V, Gonzalez R, Grob J-J, Rutkowski P, Lao CD, et al. Five-Year Survival with Combined Nivolumab and Ipilimumab in Advanced Melanoma. N Engl J Med. 2019 381:1535-1546.

2 Eggermont AM, Chiarion-Sileni V, Grob J-J, Dummer R, Wolchok JD, Schmidt H, et al: Prolonged survival in stage III melanoma with ipilimumab adjuvant therapy. N Engl J Med 375:1845-1855, 2016

3 Tarhini AA, Lee SJ, Hodi FS, Rao UNM, Cohen GI, Hamid O, et al. Phase III Study of Adjuvant Ipilimumab (3 or $10 \mathrm{mg} / \mathrm{kg}$ ) Versus High-Dose Interferon Alfa-2b for Resected High-Risk Melanoma: North American Intergroup E1609. J Clin Oncol. 2019 JCO1901381.

4 Betof Warner A, Postow MA. Another Victory for Immune Checkpoint Blockade in Melanoma: Adjuvant Ipilimumab Over Interferon. J Clin Oncol. 2019 Dec 27:JCO1902988.

5 Eggermont AMM, Blank CU, Mandala M, Long GV, Atkinson V, Dalle S, et al. Adjuvant Pembrolizumab versus Placebo in Resected Stage III Melanoma. N Engl J Med 2018;378:1789-801.

6 Weber J, Mandala M, Del Vecchio M, Gogas HJ, Arance AM, Cowey CL, et al. Adjuvant Nivolumab versus Ipilimumab in Resected Stage III or IV Melanoma. N EngI J Med 2017;377:1824-35.

7 Eggermont AMM, Blank CU, Mandala M, Long GV, Atkinson VG, Dalle S, et al . Prognostic and predictive value of AJCC-8 staging in the phase III EORTC1325/KEYNOTE-054 trial of pembrolizumab vs placebo in resected high-risk stage III melanoma. Eur J Cancer. 2019 Jul;116:148-157

8 Chaker L, Bianco AC, Jonklaas J, Peeters RP. Hypothyroidism. Lancet 2017;390:1550-62.

9 Thayakaran R, Adderley NJj, Sainsbury C, Torlinska B, Boelaert K, Sumilo D, et al. Thyroid replacement therapy, thyroid stimulating hormone concentrations, and long term health outcomes in patients with hypothyroidism: longitudinal study. BMJ. 2019 Sep 3;366:14892.

10 Wang DY, Salem JE, Cohen JV, Chandra S, Menzer $C_{2}$ Ye F, et al, Fatal Toxic Effects Associated With Immune Checkpoint Inhibitors: A Systematic Review and Meta-analysis. JAMA Oncol. 2018 1;4:17211728. 
11 Byun DJ, Wolchok JD, Rosenberg LM, Girotra M. Cancer immunotherapy - immune checkpoint blockade and associated endocrinopathies. Nat Rev Endocrinol 2017;13:195-207.

12 Bergthorsdottir R, Leonsson-Zachrisson M, Oden A, Johannsson G. Premature mortality in patients with Addison's disease: a population-based study. J Clin Endocrinol Metab. 2006 91: 48494853.

13 QuinklerM, Ekman B, Zhang P, Isidori AM, Murray RD, EU-AIR Investigators. Mortality data from the European Adrenal Insufficiency Registry-Patient characterization and associations. Clin Endocrinol $2018 \mathrm{Jul} ; 89(1): 30-35$.

14 Gastrelli G, Corona G, Mannucci E, Maggi M. Factors affecting spermatogenesis upon gonadotropin-replacement therapy: a meta-analytic study. Andrology 2014; 2(6): 794-808

15 The Practice Committee of the American Society fro Reproductive Medicine. Use of exogenous gonadotropins in anovulatory women: a technical bulletin. Fertility and Sterility 2008; 90(Suppl3) 712

16 Egg Freezing in Fertility Treatment. Trends and figures: 2010- 2016. Human Fertilisation and Embryology Authority. 201817 Akturk HK, Kahramangil D, Sarwal A, Hoffecker L, Murad MH, Michels AW. Immune checkpoint inhibitor-induced Type 1 diabetes: a systematic review and meta-analysis. Diabet Med 2019;36:1075-81.

18 Rawshani A, Rawshani A, Franzéen S, Eliasson B, Svensson A-M, Miftaraj M, et al. Mortality and Cardiovascular Disease in Type 1 and Type 2 Diabetes. N Engl J Med 2017;376:1407-18.

19 Tao B, Pietropaolo M, Atkinson M, etalschatz D, Taylor D. Estimating the cost of type 1 diabetes in the U.S.: a propensity score matching method. PLoS One. $2010 \mathrm{Jul}$ 9;5(7):e11501.

20 Gunnarsson C, Ryan MP, Marelli C, Baker ER, Stewart PM, Johannsson G, et al. Health Care Burden in Patients With Adrenal Insufficiency. J Endocr Soc. 2017 Apr 12;1(5):512-523.

21 Eggermont AMM, Kicinski M, Blank CU, Mandala M, Long GV, Atkinson V, et al., Prognostic and predictive value of immune-related adverse events among stage III melanoma patients included in the EORTC 1325/KEYNOTE-054 pembrolizumab vs placebo trial. JAMA Oncol. 2020 Jan 2. doi: 10.1001/jamaoncol.2019.5570. [Epub ahead of print] 